\title{
Subclasses of Meromorphically Multivalent Functions Defined By a Differential Operator
}

\author{
Dr. Rajkumar Anandrao Sukne \\ Dept: Applied Mathematics \\ Dilkap Research Institute of Engineering \& Mgmt Studies, Neral, Tal. Karjat. \\ rasukne@gmail.com
}

\begin{abstract}
In this chapter we have investigated and studied two new Subclasses $\sum_{\varrho \tau \zeta \xi m p}(\alpha, \beta, \eta)$ and $\sum_{\varrho \tau \varsigma \xi m p}^{+}(\alpha, \beta, \eta)$ of Meromorphic multivalent functions in the punctured disk $D$, utilizing principle of Differential sub ordinates we introduced various inclusion relationship \& properties of particular Subclasses those are defined and derived using differential operator. The outcomes which are concerned with subordination properties like Convolution of functions, the radii of starlikeness, coefficient inequalities, and convexity closure theorems etc. are derived \& studied.
\end{abstract}

Keywords: Multivalent functions, radii of starlikeness, coefficient inequalities, convexity, closure theorems.

\section{Introduction}

In this chapter we have investigated and studied two new Subclasses $\quad \sum_{\mathrm{e} \tau \zeta \xi \mathrm{mp}}(\alpha, \beta, \eta)$ and $\sum_{\mathrm{e} \tau \zeta \xi \mathrm{mp}}^{+}(\alpha, \beta, \eta) \quad$ of Meromorphic multivalent functions in the punctured disk D, The outcomes which are concerned with subordination properties like Convolution of functions, the radii of starlikeness, coefficient inequalities, and convexity closure theorems etc. are derived \& studied. We extended the well known concept of $(n, \delta)$ - neighborhood functions to the above defined Subclasses of multivalent functions. Let us assume that the class $\varpi \in \mathrm{D}$ which is Regular function belonging to $\mathrm{D}$ given by $\mathrm{D}=\{\mathrm{z} \in \mathrm{C}:|\mathrm{z}|<1\}$. $(\forall \mathrm{z}$ in $\mathrm{D})$. Consider

$\Omega=\{\mathrm{w} \in \varpi: \mathrm{w}$ at $\mathrm{z}=0$ is $0 \& \bmod \mathrm{w}(\mathrm{z})<1$,

Be the class of Schwarz functions. For $0 \leq \alpha<1$. Let us assume $\mathrm{q}(\alpha)=\mathrm{q} \in \widetilde{\mathrm{A}}:(0)=$

1 and Rep q(z) $>\alpha, \forall \mathrm{z}$ in $\mathrm{D}$.

Note that $q=q(0)$ is the popular class of Schwarz Caratheodory functions. The popular class of Schwarz's functions \& Caratheodary functions plays very much useful role in geometrical function theory of Regular function This is investigated and studied by various researchers. It's very simple to check $\forall \mathrm{q}$ in $\mathrm{q}(\alpha)$ if \& only if the following condition is satisfied

$\frac{\mathrm{p}(\mathrm{z})-\alpha}{1-\alpha} \in \mathrm{P}$

Using (1.3) and applying the properties of the functions which are in q, the lemmas given below for the above defined functions in the class $q(\alpha)$ can be obtained.

\section{Preliminary Lemmas:}

Lemma 1.1 Let us assume $p \in \varpi$. I. e. here after it is to be taken as $p \in P(\alpha) \Leftrightarrow \exists w \in \Omega$ satisfying

$q(z)=\frac{1-\langle 4(1-\eta) \propto-1\rangle w(z)}{1-w(z)}$

Lemma 1.2 (Herglotz formula): A function $p \in \varpi$ contained in the class $P(\alpha) \quad \exists$ a probability measure $\mu(y)$ on $\partial D$ satisfying the following Equation given by $q(z)=$ $\int_{|y|} \frac{1-\langle 4(1-\eta) \alpha-1\rangle y(z)}{1-y(z)} d \mu(y),(\forall z$ in $D)$.

We know that if $g<f$ i. e. here after it is to be taken as $g$ at $z=0$ is equal to 0 and $f$ at $z=0$ and $g$ (D) is subseset of $f(\mathrm{D})$. Particularly, if $f$ is univalent in disk $D$ we will get the equivalence relation $g(0)=$ $f(0)$ if \& only if $g(\mathrm{z}) \prec f(\mathrm{z}) \& g(\mathrm{D}) \subset f(\mathrm{D})$. Let us assume that $\sum_{1}$ represents the classes of all Meromorphic functions $g$ given by

$$
g(z)=\frac{1}{z^{q}}+\sum_{n=1-q}^{\infty} b_{n} z^{n} \quad\left(\forall b_{n} \geq 0, q \in N\right) .
$$

the above defined functions are multivalent Analytic in the punctured unit disk $D^{*}=D \backslash\{0\}$. given by $\sum_{q}^{+}$Represent subclass of $\sum_{q}$ which contains the functions given by

$g(z)=\frac{1}{z^{q}}+\sum_{n=1-q}^{\infty} b_{n} z^{n} \geq 0 \quad\left(\forall z\right.$ in $\left.D^{*}\right)$

Function $g \in \sum_{q}$ is said to be Meromorphic univalent starlike of order $\propto(0 \leq \propto<1)$. Where,

$-\operatorname{Rep}\left\{\frac{1}{q} \frac{z g^{\prime}(z)}{g(z)}\right\}>\propto \quad(z \in U)$.

The class $\sum_{q}^{+}(\propto)$ represents such functions that are as given above. If $g \in \sum_{q}$ is given by (1.6) \& $f \in \sum_{q}$ given below $f(z)=\frac{1}{z^{q}}+\sum_{n=1-q}^{\infty} a_{n} z^{n}$.

I. e. here after it is to be taken as the Convolution that is the $g * f$ as

$(g * f)=\frac{1}{z^{q}}+\sum_{n=1-q}^{\infty} b_{n} a_{n} z^{n} \geq 0$

$\left(\forall q\right.$ in $N \& \forall z$ in $\left.D^{*}\right)$.

For all functions $g \in \Sigma_{q}$, we are defining the differential operator $D_{\varrho \tau \varsigma \xi}^{m} \xi$ as given as follows

$D_{\varrho \tau \zeta \xi q}^{0} g(z)=g(z)$

$D_{\varrho \tau \zeta \xi q}^{1} g(z)=D_{\varrho \tau \zeta \xi q} g(z)=(\tau-\xi)(\tau-\varsigma) \frac{\left[z^{q+1} g(z)\right]^{\prime \prime}}{z^{q-1}}$

$+\frac{(\tau-\xi)-\varrho^{2}(\tau-\varsigma)}{\varrho} \frac{\left[z^{q+1} g(z)\right]^{\prime}}{z^{q}}+\left[\frac{\varrho-(\tau-\xi)-\varrho^{2}(\tau-\varsigma)}{\varrho}\right] g(z)$.

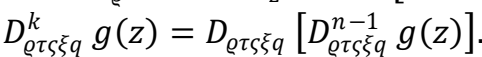

Whereit is obviously for all,

$\left(\begin{array}{c}0<\varrho \leq \frac{1}{2}, 0 \leq \xi<1, \tau \geq 1,0 \leq \varsigma<1,0 \leq \eta<1 \\ 0 \leq \alpha<1,0<\beta \leq 1 \text { and } \mathrm{k} \in \mathrm{N}\end{array}\right)$. 


\section{International Journal of Science and Research (IJSR) \\ ISSN (Online): 2319-7064}

Index Copernicus Value (2013): 6.14 | Impact Factor (2014): 5.611

If the function $f \in \Sigma_{q}$ as given in Equation (1.6) i. e. here after it is to be taken as, from (1.8) and (1.9), we have been obtained that

$D_{\varrho \tau \zeta \xi q}^{k} g(z)=\frac{1}{z^{q}}+\sum_{n=1-q}^{\infty} \Phi_{n}(\varrho, \tau, \varsigma, \xi, k, q) b_{n} z^{n}$

$\left(k\right.$ in $N, q$ in $N, z$ in $\left.D^{*}\right)$,

Here over it is,

$\Phi_{n}(\varrho, \tau, \varsigma, \xi, k, q)$

$=\left\{1+\left[(n+q)\left(\begin{array}{c}\frac{(\tau-\xi)-\varrho^{2}(\tau-\varsigma)}{\varrho} \\ +(n+q+1)(\tau-\xi)(\tau-\varsigma)\end{array}\right)\right]\right\}^{k}$.

from $(1.10) \Rightarrow D_{\varrho \tau \zeta \xi q}^{k} g(z)$ in terms of convolution can be given as follows

$D_{\varrho \tau \zeta \xi q}^{k} g(z)=(g * h)(z)$

Where it is,

$h(z)=\frac{1}{z^{q}}+\sum_{n=1-q}^{\infty} \Phi_{n}(\varrho, \tau, \varsigma, \xi, k, q) z^{n}$.

Note that, the case $\xi=\frac{1}{2} \& \tau=\varsigma$ of $D_{\varrho \tau \zeta \xi, q}^{k}$ which denotes Differential operator was investigated and studied by researchers Srivastava and Patel [60]. The differential operator tor $D_{\varrho \tau \zeta \xi p}^{m} f(z)$ for $p=1$ was considered in [50]. For the differential operator $D_{\varrho \tau \zeta \xi q}^{k} g(z)$, we are going to define a new subclass of functions given by $\sum_{q}$.

Definition 1.1 A function given by $g \in \sum_{q}$ contained in the class $\sum_{\varrho \tau \zeta \xi m q}(\alpha, \beta, \eta)$, if It will satisfy the inequality given as follows.

$\left|\frac{\frac{z\left[D_{\varrho \tau \zeta \zeta q}^{k} g(z)\right]^{\prime}}{D_{\varrho \tau \zeta \zeta q}^{k} g(z)}+2 q(1-\eta)}{\frac{z\left[D_{\varrho \tau \zeta \zeta q}^{k} g(z)\right]}{D_{\varrho \tau \zeta \zeta q}^{k} g(z)}+8 q(1-\eta)^{2} \alpha-2 q(1-\eta)}\right|<\beta$

And $\left(\begin{array}{c}0<\varrho \leq \frac{1}{2}, 0 \leq \xi<1, \tau \geq 1,0 \leq \varsigma \leq 1, \\ 0 \leq \eta<1,0 \leq \alpha<1,0<\beta \leq 1 \text { and } \forall k \text { in } N\end{array}\right)$.

Recently Mogra et.al.[62] introduced and studied given by $\sum_{\varrho \tau \zeta \xi m q}(\propto, \beta, \eta) \quad \forall \quad q=1$ and $k=0$ is the class of Meromorphic univalent starlike functions of order $\propto \&$ type $\beta$. It is simple to verify for $k=0 \& \beta=1$ the class $\sum_{\varrho \tau \zeta \xi k q}(\propto, \beta, \eta)$ reduces into the class $\sum_{q}^{*}(\propto)$. Let us assume another subclass of $\sum_{q}$ as follows

$\sum_{\varrho \tau \zeta \xi k q}^{+}(\propto, \beta, \eta)=$

$\sum_{\varrho \tau \zeta \xi k q}^{+}(\propto, \beta) \cap \sum_{\varrho \tau \zeta \xi k q}(\propto, \beta, \eta)$.

the main purpose of this chapter is to study and represent systematically investigation of the classes $\sum_{\varrho \tau \varsigma \xi k q}(\propto, \beta, \eta)$ and $\sum_{q}^{*}(\propto, \beta, \eta)$.

4.2 Properties of the Class $\sum_{\varrho \tau \varsigma \xi m p}(\propto, \beta, \eta)$.

We are going to start this part of the chapter for function which contained in given by

$\sum_{\varrho \tau \zeta \xi m p}(\propto, \beta, \eta)$ With respect required necessary \& sufficient conditions in terms of subordinations.

Theorem 2.1 A function $g \in \sum_{q}$ contained in $\sum_{\varrho \tau \zeta \xi k q}(\propto$ $, \beta, \eta)$ if $\&$ only if that will satisfy the condition as given as follows.

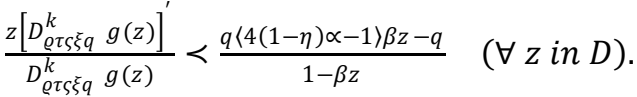

Proof Let $g \in \Sigma_{\varrho \tau \zeta \xi k q}(\propto, \beta, \eta)$. I. e. here after it is to be taken as, from (1.6), we have

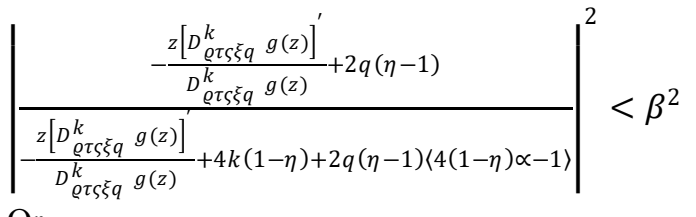

Or

$\frac{\left(1-\beta^{2}\right)}{(-\langle 4(1-\eta) \alpha-1\rangle)^{2}-1}\left|-\frac{z\left[D_{\varrho \tau \zeta \zeta q}^{k} g(z)\right]^{\prime}}{D_{\varrho \tau \zeta \xi q}^{k} g(z)}+2 q(\eta-1)\right|^{2}$

$-\frac{2\left[1+\beta^{2}(-\langle 4(1-\eta) \alpha-1\rangle)\right]}{(-\langle 4(1-\eta) \alpha-1\rangle)^{2}-1} \operatorname{Rep}\left\{-\frac{1}{2 q(1-\eta)} \cdot \frac{z\left[D_{\varrho \tau \zeta \xi q}^{k} g(z)\right]}{D_{\varrho \tau \zeta \xi q}^{k} g(z)}\right\}<\beta^{2}$

If $\beta \neq 1$, we have been obtained the relation (inequality)

$\left|-\frac{z\left[D_{\varrho \tau \zeta \zeta \xi q}^{k} g(z)\right]^{\prime}}{D_{\varrho \tau \zeta \zeta q}^{k} g(z)}-2 q(1-\eta)\right|^{2}$

$-2 \frac{1+\beta^{2}\langle 4(\eta-1) \alpha+1\rangle}{1-\beta^{2}} \operatorname{Rep}\left\{-\frac{1}{2 q(1-\eta)} \cdot \frac{z\left[D_{\varrho \tau \zeta \zeta q}^{k} g(z)\right]^{\prime}}{D_{\varrho \tau \zeta \xi q}^{k} g(z)}\right\}$

$+\left[\frac{\left.1+\beta^{2}\langle 4(\eta-1) \alpha+1\rangle\right)}{1-\beta^{2}}\right]^{2}$

$<\frac{-1-\beta^{2}\langle 4(\eta-1) \alpha+1\rangle}{1-\beta^{2}}+\left[\frac{1+\beta^{2}\langle 4(\eta-1) \alpha+1\rangle}{1-\beta^{2}}\right]^{2}$

That is,

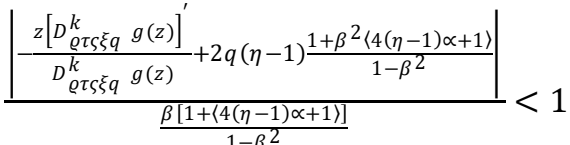

the above inequality gives the equality

$G(z)=-\frac{1}{2 q(1-\eta)} \cdot \frac{z\left[D_{\varrho \tau \zeta \zeta q}^{k} g(z)\right]^{\prime}}{D_{\varrho \tau \zeta \zeta q}^{k} g(z)}$

Which belong to the unit disk centered at $\frac{1+\beta^{2}\langle 4(\eta-1) \alpha+1\rangle}{1-\beta^{2}}$ and with the radius $\frac{\beta[1+\langle 4(\eta-1) \alpha+1\rangle]}{1-\beta^{2}}$.

Simple to verify and to check

$G(z)=\frac{1-\langle 4(1-\eta) \propto-1\rangle \beta z}{1-\beta z}$

Corresponds in the unit disk $D$ onto the disk given by

$\frac{\left|\mathrm{w}-\frac{1+\beta^{2}\langle 4(\eta-1) \alpha+1\rangle}{1-\beta^{2}}\right|}{\frac{\beta[1+\langle 4(\eta-1) \alpha+1\rangle]}{1-\beta^{2}}}<1$.

Since $G$ is Multivalent and $G(0)=F(0), G(D) \subset F(D)$, we have $F(z) \prec F(z)$, s. t.

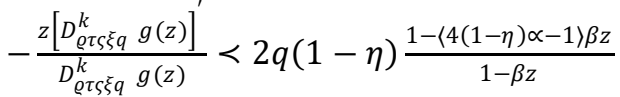

$\operatorname{Or} \frac{z\left[D_{\varrho \tau \zeta \xi q}^{k} g(z)\right]^{\prime}}{D_{\varrho \tau \zeta \xi q}^{k} g(z)}<\frac{-2 q(1-\eta)+2 q(1-\eta)\langle 4(1-\eta) \propto-1\rangle \beta z}{1-\beta z \beta z}$.

Conversely, suppose that subordination

$\frac{z\left[D_{\varrho \tau \zeta \zeta q}^{k} g(z)\right]^{\prime}}{D_{\varrho \tau \zeta \zeta q}^{k} g(z)} \prec \frac{p\langle 4(1-\eta) \propto-1\rangle \beta z-q}{1-\beta z}$ is true.

I. e. here after it is to be taken as

$-\frac{z\left[D_{\varrho \tau \zeta \zeta q}^{k} g(z)\right]^{\prime}}{D_{\varrho \tau \zeta \zeta q}^{k} g(z)}=2 q(1-\eta) \frac{1-\langle 4(1-\eta) \propto-1\rangle \beta w(z) \beta z}{1-\beta w(z) \beta z}$.

Simplifying (2.3), we get the following inequality

$$
\left|\frac{\frac{z\left[D_{\varrho \tau \zeta \xi q}^{k} g(z)\right]^{\prime}}{D_{\varrho \tau \zeta \xi q}^{k} g(z)}+2 q(1-\eta)}{\frac{z\left[D_{\varrho \tau \zeta \xi q}^{k} g(z)\right]}{D_{\varrho \varsigma \tau \xi q}^{q} g(z)}+2 q(1-\eta)\langle 4(1-\eta) \propto-1\rangle}\right|<\beta
$$

which proves that $g \in \sum_{\varrho \tau \zeta \xi k q}(\propto, \beta, \eta)$. If $\beta=1$, inequality 


\section{International Journal of Science and Research (IJSR) \\ ISSN (Online): 2319-7064}

Index Copernicus Value (2013): 6.14 | Impact Factor (2014): 5.611

(1.14) becomes

$\left|\frac{-\frac{z\left[D_{\varrho \tau \zeta \zeta q}^{k} f(z)\right]^{\prime}}{D_{\varrho \tau \zeta \zeta p}^{m} f(z)}+2 p(\eta-1)}{-\frac{z\left[D_{\varrho \tau \zeta \zeta q}^{k} g(z)\right]}{D_{\varrho \tau \zeta \zeta q}^{k} g(z)}+2 q(1-\eta)\langle 4(1-\eta) \propto-1\rangle}\right|<\beta$

From above it is simple to get the following Subordination condition

$-\frac{z\left[D_{\varrho \tau \zeta \zeta p}^{k} g(z)\right]^{\prime}}{D_{\varrho \tau \zeta \zeta q}^{k} g(z)} \prec 2 q(1-\eta) \frac{1-\langle 4(1-\eta) \propto-1\rangle z \beta z}{1-z}$

$\frac{z\left[D_{\varrho \tau \zeta \zeta p}^{k} g(z)\right]^{\prime}}{D_{\varrho \tau \zeta \zeta q}^{m} g(z)} \prec \frac{-2 q(1-\eta)+2 q(1-\eta)\langle 4(1-\eta) \propto-1\rangle 2 q(1-\eta) z}{1-z \beta z}$.

Hence the theorem proved.

Remark 2.1 Since

$\operatorname{Rep} \frac{1-\langle 4(1-\eta) \propto-1\rangle \beta z}{1-\beta z}>\propto$

It follows that

$-\operatorname{Re}\left\{\frac{1}{2 q(1-\eta)} \cdot \frac{z\left[D_{\varrho \tau \zeta \zeta q q}^{k} g(z)\right]^{\prime}}{D_{\varrho \tau \zeta \zeta q}^{k} g(z)}\right\}>\propto$.

$\therefore D_{\varrho \tau \zeta \zeta q}^{k} g(z) \in \sum_{p}^{*}(\alpha)$.

Structural formula for the class $\sum_{\varrho \tau \varsigma \xi k q}(\propto, 1, \eta)$ :

Theorem 2.2 Function $g \in \sum_{q}$ said to be in the class $\sum_{\varrho \tau \varsigma \xi \mathrm{kq}}(\propto, 1, \eta)$ if $\&$ only if $\exists$ a probability measure given by $\mu(\mathrm{y})$ on $\partial \mathrm{D}$ such as

$g(z)=\left[\frac{1}{z^{q}}+\sum_{n=1-q}^{\infty} \frac{z^{n}}{\Phi_{n}(\varrho, \tau \varsigma, \xi, k, q)}\right]$

$*\left\{z^{-q} \cdot \exp \int_{|y|} 2 q(1-\eta)[1+\langle 4(\eta-1) \propto+1\rangle] \ln (1-\right.$ $x z d \mu(y)$

Herez is in $D^{*}$. The mapping between $\sum_{\varrho \tau \varsigma \xi k q}(\propto, 1, \eta)$ and the probability measure $\mu(\mathrm{y})$ is injective mapping.

Proof In agreement with the subordination condition (2.5), we can say that

$g \in \int_{\varrho \tau \xi k q}(\propto, 1, \eta)$ If $\&$ only if

$-\frac{1}{2 q(1-\eta)} \cdot \frac{z\left[D_{\varrho \tau \zeta \zeta \xi q}^{k} g(z)\right]^{\prime}}{D_{\varrho \tau \zeta \zeta q}^{k} g(z)} \in q(\alpha)$.

From Lemma 1.2, we obtained

$-\frac{z\left[D_{\varrho \tau \zeta \zeta p}^{m} f(z)\right]^{\prime}}{D_{\varrho \tau \zeta \zeta p}^{m} f(z)}=2 q(1-\eta) \int_{|y|} \frac{1-\langle 4(1-\eta) \propto-1\rangle y z}{1-y z} d \mu(y)$

Which is equivalent to

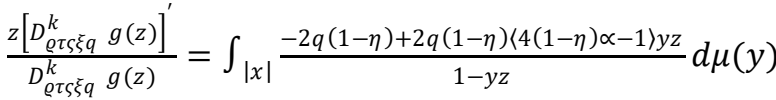

Integrating both the sides of above inequality, we have obtained

$z^{q} D_{\varrho \tau \zeta \zeta q}^{k} g(z)=\exp \int_{|y|} 2 q(1-\eta)[1+\langle 4(\eta-1) \propto$

$+1 \ln 1-y z d \mu y$.

Or

$D_{\varrho \tau \zeta \xi q}^{k} g(z)=$

$z^{-q} \cdot \exp \int_{|y|} 2 p(1-\eta)[1+\langle 4(\eta-1) \propto+1\rangle] \log (1-$

$x z d \mu(y)$

$D_{\varrho \tau \zeta \xi q}^{k} g(z)=(g * h)(z)$.

$h(z)=\frac{1}{z^{q}}+\sum_{n=1-q}^{\infty} \Phi_{n}(\varrho, \tau, \varsigma, \xi, k, q) z^{n}$
Using (2.10)

$g(z)=\left[\frac{1}{z^{q}}+\sum_{n=1-q}^{\infty} \frac{z^{n}}{\Phi_{k}(\varrho, \tau, \zeta, \xi, k, q)}\right] *\{\ln (1-y z) d \mu(y)\}$. Where it is obviously for all $\left(\forall z\right.$ in $\left.U^{*}\right)$.

Theorem 2.3 Let $g \in \sum_{\varrho \tau \varsigma \xi k q}(\propto, 1, \eta)$ i. e. here after it is to be taken as

$z^{q} D_{\varrho \tau \zeta \zeta q}^{k} g(z) \prec(1-z)^{2 q(1-\eta)[1+\langle 4(\eta-1) \propto+1\rangle]}, \quad \forall z$ in $U$.

Proof Let $g \in \sum_{\varrho \tau \varsigma \xi k q}(\propto, 1, \eta)$ i. e. here after it is to be taken as by (2.5) we have

$\frac{z\left[D_{\varrho \tau \zeta \zeta q}^{k} g(z)\right]^{\prime}}{D_{\varrho \tau \zeta \zeta q}^{k} g(z)} \prec \frac{-2 q(1-\eta)+2 q(1-\eta)\langle 4(1-\eta) \propto-1\rangle z}{1-z}$

Since the function

$\underline{-2 q(1-\eta)+2 q(1-\eta)\langle 2(1-\eta) \propto-1\rangle z}$

is convex and in $\mathrm{U}$, In agreement with Goluzin's statement we have obtained

$\int_{0}^{z} \frac{z\left[D_{\varrho \tau \zeta \zeta q}^{k} g(z)\right]}{D_{\varrho \tau \zeta \zeta q}^{k} g(z)} d \zeta \prec \int_{0}^{z} \frac{-2 q(1-\eta)+2 q(1-\eta)\langle 4(1-\eta) \propto-1\rangle \zeta}{\zeta(1-\zeta)} d \zeta$

Or

$\ln D_{\varrho \tau \zeta \zeta q}^{k} g(z) \prec \ln \frac{[1-z]^{2 q(1-\eta)[1+\langle 4(\eta-1) \alpha+1\rangle]}}{(z)^{q}}$

Thus $\exists$ a function $w \in \Omega$ s.t.

$\ln D_{\varrho \tau \zeta \xi q}^{k} g(z) \prec \ln \frac{[1-w(z)]^{2 q(1-\eta)[1+\langle 4(\eta-1) \alpha+1\rangle]}}{w(z)^{q}}$,

The above defined relation is equivalent to the subordination condition given below.

$z^{q} D_{\varrho \tau \zeta \xi q}^{k} g(z)<[1-z]^{2 q(1-\eta)[1+\langle 4(\eta-1) \propto+1\rangle]}$.

Structural formula for the class $\sum_{\varrho \tau \varsigma \xi k q}(\propto, \beta, \eta)$ :

Theorem 2.4 let $g \in \sum_{\varrho \tau \varsigma \xi k q}(\propto, \beta, \eta)$. I. e. here after it is to be taken as

$g(z)=\left[z^{-q}+\sum_{n=1-q}^{\infty} \frac{z^{n}}{\Phi_{n}(\varrho, \tau, \zeta, \xi, k, q)}\right] *$

$\left[z^{-q} \exp (2 q(1-\eta)[\beta+\right.$

$4 \eta-1 \beta \alpha+\beta 0 z w(\zeta) 1-\beta w(\zeta) d \zeta$.

Where $\left(\forall z\right.$ in $\left.D^{*}\right) \quad \& \quad(w \in \Omega)$.

Proof Let $g \in \sum_{\varrho \tau \zeta \zeta k q}(\propto, \beta, \eta)$ and since we have obtained $\frac{z\left[D_{\varrho \tau \zeta \zeta q}^{k} g(z)\right]^{\prime}}{D_{\varrho \tau \zeta \zeta q}^{k} g(z)} \prec \frac{p\langle 4(1-\eta) \propto-1\rangle \beta z-p}{1-\beta z}, \quad(\forall z$ in $D)$,

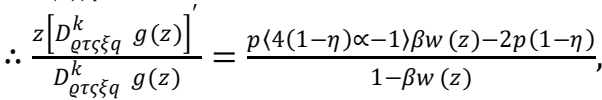

From above Equation, we have

$\frac{z\left[D_{\varrho \tau \zeta \zeta q}^{k} g(z)\right]^{\prime}}{D_{\varrho \tau \zeta \zeta q}^{k} g(z)}+\frac{2 p(1-\eta)}{z}=\frac{2 q(1-\eta)\langle 4(1-\eta) \propto-1\rangle \beta w(z)-2 q(1-\eta)}{1-\beta w(z)}$,

Integrating above implies that

$\frac{\ln \left[z^{q} D_{\varrho \tau \zeta \zeta q}^{k} g(z)\right]}{2 q(1-\eta)[1+\langle 4(\eta-1) \propto+1\rangle]}=\beta \int_{0}^{z} \frac{w(\zeta)}{[1-\beta w(\zeta)]} d \zeta$.

$D_{\varrho \tau \zeta \zeta q}^{k} g(z)=(g * h)(z)$,

Where it is obviously for all,

$h(z)=z^{-q}+\sum_{n=1-q}^{\infty} \Phi_{n}(\varrho, \tau, \varsigma, \xi, k, q) z^{n}$.

Using (2.10)

$g(z)=\left[z^{-q}+\sum_{n=1-q}^{\infty} \frac{z^{n}}{\Phi_{n}(\varrho, \tau, \zeta, \xi, k, q)}\right] *$ 


\section{International Journal of Science and Research (IJSR) \\ ISSN (Online): 2319-7064}

Index Copernicus Value (2013): 6.14 | Impact Factor (2014): 5.611

$\left[z^{-q} \exp (2 q(1-\eta)[1+\right.$

$4 \eta-1 \alpha+1 \beta 0 z w(\zeta) 1-\beta w(\zeta) d \zeta$.

Theorem 2.5 If function $g \in \Sigma_{q}$ is said to be in the class $\Sigma_{\varrho \tau \varsigma \xi m p}(\propto, \beta, \eta)$, i. e. here after it is to be taken as $D_{\varrho \tau \zeta \xi q}^{k} g(z)$

$*\left\{\begin{array}{c}\frac{-2 q(1-\eta) z^{-q}+(q+1) z^{1-q}}{(1-z)^{2}}\left(1-\beta e^{i \theta}\right) \\ +\frac{z^{-q}}{(1-z)}\left[2 q(1-\eta)-2 q(1-\eta)\langle 4(1-\eta) \propto-1\rangle \beta e^{i \theta}\right]\end{array}\right\}$

$\neq 0 \quad \forall z$ in $D^{*} \& \quad \theta \in(0,2 \pi)$.

Proof Let $g \in \sum_{\varrho \tau \zeta \xi k q}(\propto, \beta, \eta)$. I. e. here after it is to be taken as, from (2.1) gives the following

$$
-\frac{z\left[D_{\varrho \tau \zeta \zeta q}^{k} g(z)\right]^{\prime}}{D_{\varrho \tau \zeta \zeta q}^{k} g(z)} \neq \frac{-2 q(1-\eta)\langle 4(1-\eta) \propto-1\rangle \beta e^{i \theta}+q}{1-\beta e^{i \theta}}
$$

$\forall z$ in $D, \theta \in(0,2 \pi)$

It is simple to show that the above condition (2.12) is equivalent to the following

$\left(1-\beta e^{i \theta}\right) z\left[D_{\varrho \tau \zeta \zeta q}^{k} g(z)\right]^{r i \theta} D_{\varrho \tau \zeta \zeta q}^{k} g(z) \neq 0$

$\therefore D_{\varrho \tau \zeta \xi q}^{k} g(z)=D_{\varrho \tau \zeta \xi q}^{k} g(z) *\left(z^{-q}+z^{1-q}+\cdots+\frac{1}{z}+1+\right.$ $z 1-z=D \varrho \tau \varsigma \xi q k g(z) * z-q 1-Z$

(2.14)

And $z\left[D_{\varrho \tau \zeta \zeta q}^{k} g(z)\right]^{\prime}=$

$D_{\varrho \tau \zeta \zeta q}^{k} g(z) *\left(-q z^{-q}+(1-q) z^{1-q}-\cdots \cdot-\frac{1}{z}+\frac{z}{(1-z)^{2}}\right)=$

$D_{\varrho \tau \zeta \zeta q}^{k} g(z) * \frac{-q z^{-q}+(1+p) z^{1-q}}{(1-z)^{2}}$

$-\frac{z\left[D_{\varrho \tau \zeta \zeta q}^{k} g(z)\right]^{\prime}}{D_{\varrho \tau \zeta \zeta q}^{k} g(z)} \neq \frac{-2 q(1-\eta)\langle 4(1-\eta) \propto-1\rangle \beta e^{i \theta}+q}{1-\beta e^{i \theta}}$. Where

$\forall z$ in $D, \theta \in(0,2 \pi)$.

\section{Coefficient Estimates}

Theorem 2..6 Let function $g$ be of the form (1.6) contained in the class $\sum_{\varrho \tau \zeta \xi k q}(\propto, \beta, \eta)$.I. e. here after it is to be taken as, for $m \geq 3-2 q(1-\eta)$

$\frac{\left|a_{m}\right|(m+q) \Phi_{m}(\varrho, \tau, \varsigma, \xi, k, q)}{2 q(1-\eta)[1+\langle 4(\eta-1) \alpha+1\rangle]} \leq \beta$.

here $\Phi_{m}(\varrho, \tau, \xi, k, q)$ is given by $(1.11)$

Proof We use the method of Koegh and Clunie [12] to prove the above mentioned Coefficient Estimates given by (2.16). For $g \in \sum_{\varrho \tau \zeta \xi k q}(\propto, \beta, \eta)$, we

have

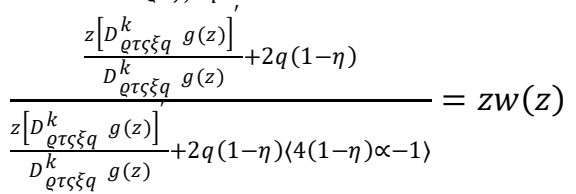

where $w$ is Analytic function in the unit $D \&|w(z)| \leq \beta$ $\forall z$ in $D$. I. e. here after it is to be taken as $z\left[D_{\varrho \tau \zeta \zeta q}^{k} g(z)\right]+q D_{\varrho \tau \zeta \xi q}^{k} g(z)=$

$z w(z)\left[\begin{array}{c}z\left(D_{\varrho \tau \zeta \xi q}^{k} g(z)\right)^{\prime} \\ +2 q(1-\eta)\langle 4(1-\eta) \propto-1\rangle D_{\varrho \tau \zeta \xi q}^{k} g(z)\end{array}\right]$.

$z w(z)=\sum_{n=1}^{\infty} w_{n} z^{n}$,

Using the equations (1.10) \& (2.17), here we are going to obtain

$\sum_{n=1-q}^{\infty}(n+q) \Phi_{n}(\varrho, \tau, \varsigma, \xi, k, q) a_{n} z^{n+q}=$

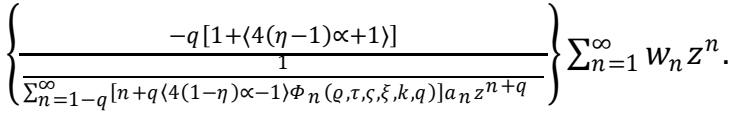

By using above Equation (2.18), we have obtained $m \Phi_{m-q}(\varrho, \tau, \varsigma, \xi, k, q) a_{m-q}$

$=-2 q(1-\eta)[1+\langle 4(\eta-1) \propto+1\rangle] w_{m}$,

For $\mathrm{m}=1,2$

And

$m \Phi_{m-q}(\varrho, \tau, \varsigma, \xi, k, q) a_{m-q}=-2 q(1-\eta)[1+$

$4 \eta-1 \alpha+1 w m \quad+n=1-q m-1-q n+2 q 1-\eta 21-\eta \alpha-1$

Фnanwm-q-n $\forall m \geq 3$

From (2.19), we obtain

$\left\{\begin{array}{c}-4 q(1-\eta)(1-\alpha)+ \\ \sum_{n=1-q}^{m-1-q}\left[n+2 q(1-\eta)\langle 4(1-\eta) \propto-1\rangle \Phi_{n}\right] a_{n} z^{n+q}\end{array}\right\}$

$\times \sum_{n=1}^{\infty} w_{n} z^{n}$

$=\sum_{n=1-q}^{m-q}\left[(n+q) \Phi_{n}(\varrho, \tau, \varsigma, \xi, k, q)\right] a_{n} z^{n+q}$

$+\sum_{n=1-q}^{m-1-q} c_{n} z^{n+q}$

It is known that, if $h(z)=\sum_{n=1-q}^{m-1-q} h_{m} z^{m}$ i. e. here after it is to be taken as for $0<r<1$,

$\sum_{m=0}^{\infty}\left|h_{m}\right|^{2} r^{2 m}=\frac{1}{2 \pi} \int_{0}^{2 \pi}\left|h\left(r e^{i i \theta}\right)\right|^{2} d \theta$

$\therefore$ the following

$\left|\sum_{n=1}^{\infty} w_{n} z^{n}\right|<\beta|z|<\beta$,

Using (2.20) and (2.21), we have been obtained the relation

$\sum_{n=1-q}^{m-q}[(n+$

$2 q 1-\eta 2 \Phi n \varrho, \tau, \varsigma, \xi, k, q 2 a n 2 r 2(n+q)+$

$\sum_{n=m+1-q}^{\infty}\left|c_{n}\right|^{2} r^{2(n+q)} \leq \beta^{2}\left\{q^{2}[1+\langle 4(\eta-1) \propto+1\rangle]^{2}\right.$

$+\sum_{n=1-q}^{m-1-q}[n+$

$2 q 1-\eta 41-\eta \alpha-12 \Phi n \varrho, \tau, s, \xi, k, q 2 a n 2 r 2 n+q$

Letting $r \rightarrow 1$, we have been obtained the relation $\sum_{n=1-q}^{m-1-q}[n+2 q(1-\eta)]^{2}\left(\Phi_{n}(\varrho, \tau, \varsigma, \xi, k, q)\right)^{2}\left|a_{n}\right|^{2}$ $\leq 4(1-\eta)^{2} p^{2} \beta^{2}[1+\langle 4(\eta-1) \propto+1\rangle]^{2}$

$+\sum_{n=1-q}^{m-1-q}[n+$

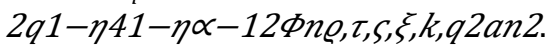

The above relation (inequality) implies

$m^{2} \Phi_{m-q}(\varrho, \tau, \varsigma, \xi, k, q)^{2}\left|a_{m-q}\right|^{2}$

$\leq 4(1-\eta)^{2} q^{2} \beta^{2}[1+\langle 4(\eta-1) \propto+1\rangle]^{2}$

$+\sum_{n=1-q}^{m-1-q}[k+2 q(1-\eta)]^{2}\left(\Phi_{n}(\varrho, \tau, \varsigma, \xi, k, q)\right)^{2}\left|a_{n}\right|^{2}$

Finally, replacing $\mathrm{m}-\mathrm{q}$ by $\mathrm{m}$, we have

$\left|a_{m}\right| \leq \frac{4 q(1-\eta) \beta\left[\frac{1+\langle 4(\eta-1) \propto+1\rangle}{2}\right]}{(m+2 q(1-\eta)) \Phi_{n}(\varrho, \tau, \zeta, \xi, k, q)}$

Hence above theorem is proved. Now theorem 2.6 given by the class $\sum_{\varrho \tau \zeta \xi m p}(\propto, \beta, \eta)$.

Corollary 2.1 If $g \in \sum_{\varrho \tau \zeta \xi k q}(\alpha, \beta, \eta)$ is given by (1.6), i. e. here after it is to be taken as $\forall z: 0<|z|=r<1$ $|g(z)| \leq$ $\frac{1}{r^{q}}+$

$q \beta[1+\langle 4(\eta-1) \propto+1\rangle] r^{1-q} \sum_{n=1-q}^{\infty} \frac{1}{(n+q) \Phi_{n}(\varrho, \tau \varsigma, \xi, k, q)}$.

$|g(z)| \geq \frac{1}{r^{q}}+2 q(1-\eta) \beta[1+\langle 4(\eta-1) \propto+1\rangle]$ 


\section{International Journal of Science and Research (IJSR) \\ ISSN (Online): 2319-7064}

Index Copernicus Value (2013): 6.14 | Impact Factor (2014): 5.611

$\times r^{1-q} \sum_{n=1-q}^{\infty} \frac{1}{(n+2 q(1-\eta)) \Phi_{n}(\varrho, \tau, \zeta, \xi, k, q)}$.

And

$\left|g^{\prime}(z)\right| \geq \frac{2 q(1-\eta)}{r^{q}+1}-2 q(1-\eta) \beta[1+\langle 4(\eta-1) \propto+1\rangle]$

$\times r^{2-q} \sum_{n=1-q}^{\infty} \frac{1}{(n+2 q(1-\eta)) \Phi_{n}(\varrho, \tau, \varsigma, \xi, k, q)}$.

$\left|g^{\prime}(z)\right| \leq \frac{2 q(1-\eta)}{r^{q+1}}+4 q(1-\eta)^{2} \beta[1+\langle 4(\eta-1) \propto+1\rangle]$

$\times r^{2-q} \sum_{n=1-q}^{\infty} \frac{1}{(n+2 q(1-\eta)) \Phi_{n}(\varrho, \tau, \zeta, \xi, k, q)}$.

It will contained in the class given as

$\sum_{\varrho \tau \zeta \xi m p}(\propto, \beta, \eta)$. which is sufficient condition

Theorem 2.7 Let us assume that $g \in \sum_{p}$ represented by (1.6).

If $\forall \propto(0 \leq \alpha<1)$

and $\forall \beta(0<\beta \leq 1)$

$\sum_{n=1-q}^{\infty}\left\{\begin{array}{c}n(\beta+1)+ \\ 2 q(1-\eta)[1+\beta\langle 4(1-\eta) \propto-1\rangle]\end{array}\right\} \times$

on $, \tau, \xi, k, q$ an

$\leq q(1-\eta) \beta[1+\langle 4(\eta-1) \propto+1\rangle]$

Where it is, $g \in \sum_{\varrho \tau \varsigma \xi k q}(\propto, \beta, \eta)$.

Proof We assume $g(z)=z^{-q}+\sum_{n=1-q}^{\infty} a_{n} z^{n}$.

We get

$M=\left|z\left[D_{\varrho \tau \zeta \xi q}^{k} g(z)\right]^{\prime}+2 p(1-\eta) D_{\varrho \tau \zeta \xi q}^{k} g(z)\right|$

$-\beta \mid z\left[D_{\varrho \tau \zeta \xi q}^{k} g(z)\right]^{\prime}+$

$2 q 1-\eta 41-\eta \alpha-1 D \varrho \tau \varsigma \xi q k g(z)$

$=\mid \sum_{n=1-q}^{\infty}(n+2 q(1-\eta)) \Phi_{n}(\varrho, \tau, \varsigma, \xi, k, q) a_{n} z^{n}$

$-\beta\left|\frac{-2 q(1-\eta)[1+\langle 4(\eta-1) \propto+1\rangle]}{z^{q}}\right|+\sum_{n=1-q}^{\infty}[n+2 q(1-$

$\eta 41-\eta \alpha-1 \Phi n \varrho, \tau, \varsigma, \xi, k, q a n z n$

For $0<|z|=r<1$, we have been obtained the following inequality

$r^{q} M \leq \sum_{n=1-q}^{\infty}(n+q) \Phi_{n}(\varrho, \tau, \varsigma, \xi, k, q) a_{n} r^{n+q}$

$-\beta\left[\begin{array}{c}2 q(\eta-1)(\langle 4(\eta-1) \propto+1\rangle+1) \\ -\sum_{n=1-q}^{\infty}|n+2 q(1-\eta)\langle 4(1-\eta) \propto-1\rangle| \Phi_{n} a_{n} r^{n+q}\end{array}\right]$.

Or $r^{q} M \leq \sum_{\mathrm{n}=1-\mathrm{q}}^{\infty}\{\mathrm{n}(\beta+1)+2 \mathrm{q}(1-\eta)[1+$

$\beta 41-\eta \propto-1$

$\times \Phi_{\mathrm{n}}\left|\mathrm{a}_{\mathrm{n}}\right| \mathrm{r}^{\mathrm{n}+\mathrm{q}}-2 \mathrm{q}(1-\eta) \beta[1+\langle 4(\eta-1) \propto+1\rangle]$.

Because of the inequality mentioned above holds

$\forall r(0<r<1)$, letting $\mathrm{r} \rightarrow 1$, we have obtained

$M \leq \sum_{n=1-q}^{\infty} n(\beta+1)+2 q(1-\eta)[1+\beta\langle 4(1-\eta) \propto$

$-1 \times$ Фnan $-2 q 1-\eta \beta 1+4 \eta-1 \alpha+1$.

Using the Equation (2.22), we have obtained $\mathrm{M} \leq 0$, $\quad \therefore$

$\left|\frac{z\left[D_{\varrho \tau \zeta \zeta q}^{k} g(z)\right]^{\prime}}{D_{\varrho \tau \zeta \zeta q}^{k} g(z)}+2 p(1-\eta)\right|<\beta \mid \frac{z\left[D_{\varrho \tau \zeta \zeta q}^{k} g(z)\right]^{\prime}}{D_{\varrho \tau \zeta \zeta q}^{k} g(z)}+$

$2 q 1-\eta 41-\eta \alpha-1$.

Consequently, $g \in \sum_{\varrho \tau \varsigma \xi k q}(\propto, \beta, \eta)$.

\section{Properties of the class $\sum_{\varrho \tau \varsigma \xi m p}^{+}(\propto, \boldsymbol{\beta}, \boldsymbol{\eta})$ :}

In this part of the chapter we are proving that $\forall$ functions which are in the class $(2.22) \sum_{\varrho \tau \zeta \xi m p}^{+}(\propto, \beta, \eta)$ the Equation (2.22) is required necessary and sufficient condition.

Theorem 3.1 Let us assume a function $g \in \sum_{q}^{+}$, i. e. here after it is to be taken as $\forall g$ in the class

$\sum_{\varrho \tau \zeta \xi m p}^{+}(\propto, \beta, \eta)$ If $\&$ only if $\sum_{n=1-q}^{\infty}\{n(\beta+1)+2 q(1-\eta)[1+\beta\langle 2(1-\eta) \propto-1\rangle]\}$

$\times \Phi_{n}(\varrho, \tau, \varsigma, \xi, k, q) a_{n} \leq 2 q(1-\eta) \beta[1+\langle 4(\eta-1) \propto$

+1 .

Proof In agreement with result obtained as 2.7, we are proving that "only if" part. Consider that $g(z)=z^{-q}+\sum_{n=1-q}^{\infty} a_{n} z^{n} \quad\left(a_{n} \geq 0, q\right.$ in $\left.N\right)$ is in the class $\sum_{\varrho \tau \zeta \xi k q}^{+}(\propto, \beta, \eta)$.

$\therefore\left|\frac{\frac{z\left[D_{\varrho \tau \zeta \zeta q}^{k} g(z)\right]}{D_{\varrho \tau \zeta \zeta q}^{k} g(z)}+2 q(1-\eta)}{\frac{z\left[D_{\rho \tau \zeta \zeta q}^{k} g(z)\right]}{D_{\varrho \tau \zeta \zeta q}^{k} g(z)}+2 q(1-\eta)\langle 4(1-\eta) \propto-1\rangle}\right|=$

$\left|\frac{\sum_{n=1-q}^{\infty}(k+2 q(1-\eta)) \Phi_{n}(\varrho, \tau, \zeta, \xi, k, q) a_{n} r^{n+q}}{\frac{2 q(1-\eta)[1+\langle 4(\eta-1) \alpha+1\rangle]}{z^{q}}-\sum_{n=1-q}^{\infty}[n+2 q(1-\eta)\langle 4(1-\eta) \propto-1\rangle] \Phi_{n} a_{n} z^{n}}\right|<$

$\beta$

$\forall z$ in $D . \therefore \operatorname{Rep} z \leq|z|$. It is obvious that

$\operatorname{Re}\left\{\frac{\sum_{n=1-q}^{\infty}(n+2 q(1-\eta)) \Phi_{n}(\varrho, \tau, s, \xi, k, q) a_{n} r^{n+q}}{\frac{2 q(1-\eta)[1+(4(\eta-1) \alpha+1\rangle]}{z q}-\sum_{n=1-q}^{\infty}[n+2 q(1-\eta)\langle 4(1-\eta) \propto-1)] \Phi_{n} a_{n} z^{n}}\right\}$

$<\beta$. (3.1)

take the values of $\mathrm{z}$ on $\mathrm{x}$-axis of $\mathrm{z}$-plane s. t.

$\frac{1}{2 q(1-\eta)} \cdot \frac{z\left[D_{\varrho \tau \zeta \zeta q}^{k} g(z)\right]^{\prime}}{D_{\varrho \tau \zeta \zeta q}^{k} g(z)}$

If we clear the denominator in the Equation (2.9.1) and consider as $z \rightarrow 1$ all positive values $i$. e. here after it is to be taken as we get

$\sum_{n=1-q}^{\infty}(n+2 q(1-\eta)) \Phi_{n}(\varrho, \tau, \varsigma, \xi, k, q) a_{n}$

$\leq 2 q(1-\eta) \beta[1+\langle 4(\eta-1) \propto+1\rangle]-\sum_{n=1-q}^{\infty} \beta[n+$

$2 q 1-\eta 41-\eta \alpha-1 \Phi n \varrho, \tau, \varsigma, \xi, k, q$ an

Or

$\sum_{n=1-q}^{\infty}\{n(\beta+1)+2 q(1-\eta)[1+\beta\langle 4(1-\eta) \propto$

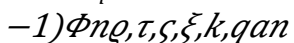

$\leq q \beta[1+\langle 4(\eta-1) \propto+1\rangle]$

Corollary 3.1 If a function $g \in \sum_{q}^{+}$defined as in equation (1.7) contained in the class $\sum_{\varrho \tau \zeta \xi m p}^{+}(\propto, \beta, \eta)$ i. e. here after it is to be taken as we say that for $n \geq 1-2 q(1-\eta)$.

$\mathrm{b}_{\mathrm{n}} \leq \frac{2 \mathrm{q}(1-\eta) \beta[1+\langle 4(\eta-1) \alpha+1\rangle]}{\{\mathrm{n}(\beta+1)+2 \mathrm{q}(1-\eta)[1+\beta\langle 4(1-\eta) \alpha-1\rangle]\} \Phi_{n}(\varrho, \tau, \zeta, \xi, k, q)}$

for the functions given below the equality

$g_{n}(z)=\frac{1}{z^{q}}-\frac{2 q(1-\eta) \beta[1+\langle 4(\eta-1) \alpha+1\rangle]}{\{n(\beta+1)+2 q(1-\eta)[1+\beta\langle 4(1-\eta) \alpha-1\rangle]\} \Phi_{n}(\varrho, \tau, \zeta, \xi, k, q)} z^{n}$

Corol. 3.1 gives a distortion result for $\sum_{\varrho \tau \zeta \xi k q}^{+}(\propto, \beta, \eta)$.

Theorem 3.2If a function $g \in \sum_{\varrho \tau \zeta \xi m p}^{+}(\alpha, \beta, \eta)$, i. e. here after it is to be taken as $\forall z(0<|z|<1)$

$|g(z)| \geq$

$\frac{1}{z^{p}}-\frac{2 q(1-\eta) \beta[1+\langle 4(\eta-1) \propto+1\rangle]}{\{\beta[1-2 q(1-\eta)+\langle 4(1-\eta) \propto-1\rangle 2 q(1-\eta)]+1\} \Phi_{1-q}(\varrho, \tau, \varsigma, \xi, k, q)} r^{1-q}$

$|g(z)| \geq$

$\frac{1}{z^{q}}+\frac{2 q(1-\eta) \beta[1+\langle 4(\eta-1) \propto+1\rangle]}{\{\beta[1-2 q(1-\eta)+\langle 4(1-\eta) \propto-1\rangle 2 q(1-\eta)]+1\} \Phi_{1-q}(\varrho, \tau, \varsigma, \xi, k, q)} r^{1-q}$.

Where the inequality holds true for the functions given as

$g_{q}(z)=$

$\frac{1}{z^{q}}+\frac{2 q(1-\eta) \beta[1+\langle 4(\eta-1) \alpha+1\rangle]}{\{\beta[1-2 q(1-\eta)+\langle 4(1-\eta) \alpha-1\rangle 2 q(1-\eta)]+1\} \Phi_{1-q}(\varrho, \tau, \zeta, \xi, k, q)} z^{1-q}$

at $z=i r, r$.

Proof Supposing $g \in \sum_{\varrho \tau \zeta \xi k q}^{+}(\propto, \beta, \eta)$ and using the inequality given as

$\sum_{n=1-q}^{\infty} b_{n} \leq$ 


\section{International Journal of Science and Research (IJSR) \\ ISSN (Online): 2319-7064}

Index Copernicus Value (2013): 6.14 | Impact Factor (2014): 5.611

$2 q(1-\eta) \beta[1+\langle 4(\eta-1) \propto+1\rangle]$

$\overline{\{\beta[1-2 q(1-\eta)+\langle 4(1-\eta) \alpha-1\rangle 2 q(1-\eta)]+1\} \Phi_{1-q}(\varrho, \tau, \zeta, \xi, k, q)}$

this by theorem 3.1, the proof of the given theorem. In the next part we are proving that the class $\sum_{\mathrm{\rho \tau} \varsigma \xi \mathrm{kq}}^{+}(\propto, \beta, \eta)$ is closed under convolution.

Theorem 3.3 Let us assume that function $h(z)$ which is Analytic in $U^{*}$ and given $h(z)=z^{-q}+\sum_{n=1-q}^{\infty} c_{n} z^{n}$ implies that $\quad 0 \leq c_{\mathrm{n}} \leq 1$. If function $g$ given in (1.7) is in the class $\sum_{\varrho \tau \varsigma \xi k q}^{+}(\propto, \beta, \eta)$ i. e. here after it is to be taken as the convolution of functions $g \& h(g * h)$ contained in $\sum_{\varrho \tau \zeta \xi m p}^{+}(\propto, \beta, \eta)$.

Proof Since $f \in \sum_{\varrho \tau \xi m p}^{+}(\propto, \beta, \eta)$, i. e. here after it is to be taken as by Theorem 3.1,

$$
\begin{gathered}
\sum_{n=1-q}^{\infty}\{n(\beta+1)+ \\
2 q 1-\eta 1+\beta 41-\eta \alpha-1 \Phi n \varrho, \tau, \varsigma, \xi, k, q b k \\
\leq 2 q(1-\eta) \beta[1+
\end{gathered}
$$

$4 \eta-1 \alpha+1$

In agreement with the inequality given as above relation $\&$ the result given below

$(g * h)(z)=z^{-q}+\sum_{n=1-q}^{\infty} b_{n} c_{n} z^{n}$

$\therefore$

$\sum_{n=1-q}^{\infty}\left\{\begin{array}{c}n(\beta+1)+ \\ 2 q(1-\eta)[1+\beta\langle 4(1-\eta) \propto-1\rangle]\end{array}\right\} \Phi_{n}(\varrho, \tau, \varsigma, \xi, k, q) b$

$1+2 q 1-\eta 1+\beta 41-\eta \alpha-1 \Phi n \varrho, \tau, s, \xi, k, q b k$

$\leq 2 q(1-\eta) \beta[1+\langle 4(\eta-1) \propto+1\rangle]$.

Theorem 3.4 If the function $g$ defined as $g \in$

$\sum_{\varrho \tau \zeta \xi k q}^{+}(\propto, \beta, \eta)$, and the integral operator as given below

$G_{c, q}(z)=\frac{c}{z^{p+c}} \int_{0}^{z} t^{c+2 p(1-\eta)+1} f(t) d t, c>0$.

Contained in $\sum_{\varrho \tau \varsigma \xi m p}^{+}(\propto, \beta, \eta)$.

Proof it is very simple to understand and simple to verify \& check that

$F_{c, p}(z)=f(z) *\left(z^{-p}+\sum_{k=1-p}^{\infty} \frac{c}{c+2 q(1-\eta)+k} z^{k}\right)$.

$\therefore 0<\frac{c}{c+2 q(1-\eta)+k} \leq 1$,

Theorem holds true.

\section{Neighborhoods and Partial Sums}

The well-known $(n, \delta)-\quad$ neighborhoods of Regularfunctions \& partial sums was earlier investigated and studied by many authors Aouf [71], Goodman [19], [20], [21], Orhan [46], Ruscheweyh [56], [59], Liu [54], Altina [3], [46], [58], Deniz and Orhan [14] and more recently by Liu and Srivastava [38]. We have defined the $(n, \delta)-$ neighborhoods of Analytic functions $g \in \sum_{1}$ of the form (2.7.6).

Definition 4.1 $\forall, \quad \delta=\frac{2(\tau-\xi)^{2}+\frac{1}{\varrho}(\tau-\xi)\left(1-\varrho^{2}\right)}{1+2(\tau-\xi)^{2}+\frac{1}{\varrho}(\tau-\xi)\left(1-\varrho^{2}\right)}>0$

$\&$ non negative sequence $s=\left\{s_{n}\right\}_{n=1-q}^{\infty}$

Where it is obviously for,

$$
s_{n}=
$$

$\underline{\{\beta[1-2 q(1-\eta)+|\langle 4(1-\eta) \propto-1\rangle| 2 q(1-\eta)]+1\} \Phi_{1-q}(\varrho, \tau, \varsigma, \xi, k, q)}$

$$
2 q(1-\eta) \beta[1+\langle 4(\eta-1) \propto+1\rangle]
$$

The $(n, \delta)-$ neighborhoods of Analytic functions $g \in \sum_{q}$ given by (1.6) defined as

$N_{\delta}(g)=\left\{\begin{array}{c}g \in \sum_{q}: g(z)=z^{-q}+\sum_{n=1-q}^{\infty} b_{n} z^{n} \text { and } \\ \sum_{n=1-q}^{\infty} S_{n}\left|b_{n}-a_{n}\right| \leq \delta\end{array}\right\}$.

$\forall S_{n}=n$, definition 1.4 corresponds to the $(n, \delta)-$ neighborhoods of analytic functions considered by Ruschewyh [56]. Using definition 4.1, we are proving results for $(n, \delta)-$ neighborhoods of Analytic functions of the class $\sum_{\varrho \tau \varsigma \xi k q}(\propto, \beta, \eta)$.

Theorem 4.1 let $g \in \sum_{\varrho \tau \zeta \xi k q}(\propto, \beta, \eta)$ denoted by Equation (1.6). If a function $g$ satisf $y$ the following relation

$\left[g(z)+\varepsilon Z^{q}\right](1+\varepsilon)^{-1} \in \sum_{\varrho \tau \zeta \xi m p}(\propto, \beta, \eta)$

$(\varepsilon \in C,|\varepsilon|<\delta, \delta \geq 0)$

i. e. here after it is to be taken as

$N_{\delta}(f) \subset \sum_{\varrho \tau \zeta \xi m p}(\propto, \beta, \eta)$

Proof $g \in \sum_{\varrho \tau \varsigma \zeta m p}(\propto, \beta, \eta)$ if \& only if

$\frac{z\left[D_{\varrho \tau \zeta \zeta q}^{k} g(z)\right]+2 q(1-\eta) D_{\varrho \tau \zeta \zeta q}^{k} g(z)}{\beta z\left[D_{\varrho \tau \zeta \zeta q}^{k} g(z)\right]^{\prime}+\beta\langle 4(1-\eta) \propto-1\rangle 2 q(1-\eta) D_{\varrho \tau \zeta \zeta q}^{k} g(z)} \neq \sigma$

$(\forall z$ in $D, \sigma \in C,|\sigma|=1)$

Equivalent to the following

$\frac{(g * h)(z)}{\nabla^{\circ}} \neq 0 \quad(\forall z$ in $D)$

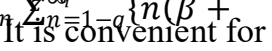

$h(z)=z^{-q}+\sum_{k=1-q}^{\infty} c_{n} z^{n}$

$=z^{-q}+$

$\sum_{n=1-q}^{\infty} \frac{\{\beta \sigma[n+\langle 4(1-\eta) \propto-1\rangle 2 q(1-\eta)]-(n+2 q(1-\eta))\} \Phi_{n}(\varrho, \tau, \varsigma, \xi, k, q)}{2 q(1-\eta) \beta[1+\langle 4(\eta-1) \propto+1\rangle] \sigma} Z^{n}$

(4.7)

From (4.7) it is oblivious that

$\left|c_{n}\right|=\left|\frac{\{\beta \sigma[n+\langle 4(1-\eta) \propto-1\rangle 2 q(1-\eta)]-(n+2 q(1-\eta))\} \Phi_{n}(\varrho, \tau, \zeta, \xi, k, q)}{2 q(1-\eta) \beta[1+\langle 4(\eta-1) \propto+1\rangle] \sigma}\right|$

$\leq \frac{\{\beta \sigma[n+|\langle 4(1-\eta) \alpha-1\rangle| 2 q(1-\eta)]+(n+2 q(1-\eta))\} \Phi_{n}(\varrho, \tau, \zeta, \xi, k, q)}{2 q(1-\eta) \beta[1+\langle 4(\eta-1) \alpha+1\rangle] \sigma}$

Further in accordance with (4.3), and by using (4.6) we have obtained the following result $\frac{\left[g(z)+\varepsilon z^{q}\right](1+\varepsilon)^{-1} * h(z)}{z^{-q}} \neq 0$.

$\operatorname{Or} \frac{(g * h)(z)}{z^{-q}} \neq \varepsilon,(\forall z$ in $D)$. which is equivalent to the result given below

$\left|\frac{(g * h)(z)}{z^{-q}}\right| \geq \delta$

Let us now consider

$f(z)=z^{-q}+\sum_{n=1-q}^{\infty} a_{n} z^{n} \in N_{\delta}(g)$,

i. e. here after it is to be taken as we have obtained

$\left|\frac{[g(z)-f(z)] *(z)}{z^{-q}}\right|=\left|\sum_{n=1-q}^{\infty}\left(b_{n}-a_{n}\right) z^{n+q}\right|$

$\leq \sum_{n=n-q}^{\infty} \frac{\{\beta[n+|\langle 4(1-\eta) \propto-1\rangle| 2 q(1-\eta)]+(n+2 q(1-\eta))\} \Phi_{n}(\varrho, \tau, \zeta, \xi, k, q)}{2 q(1-\eta) \beta[1+(4(\eta-1) \alpha+1)]}$

$\left|b_{n}-a_{n}\right||z|^{n+q}<\delta$

$(\forall z$ in $D, n \geq 1-q, q \in N, \delta>0)$.

Definition 4.2 $\forall, \delta>0$ for a non -ve sequence

$S=\left\{s_{n}\right\}_{n=1-q}^{\infty}$

Here it is obviously for,

$S_{n}=\frac{\{n(\beta+1)+2 n(1-\eta)[1+\beta\langle 4(1-\eta) \alpha-1\rangle]\} \Phi_{n}(\varrho, \tau, \zeta, \xi, k, q)}{2 q(1-\eta) \beta[1+\langle 4(\eta-1) \alpha+1\rangle]}$

$(n \geq 1-q, q \in N, 0 \leq \propto<1,0<\beta \leq 1)$

The $(n, \delta)-$ neighborhoods holo morphic functions $g \mathrm{~s}$.

$(n \geq 1-q, q \in N, 0 \leq \alpha<1,0<\beta \leq 1)$. 


\section{International Journal of Science and Research (IJSR) \\ ISSN (Online): 2319-7064}

Index Copernicus Value (2013): 6.14 | Impact Factor (2014): 5.611

t. $g \in \sum_{q}^{+}$as given in (1.7) which is given by

$N_{\delta}(g)=\left\{\begin{array}{c}f \in \sum_{q}^{+}: f(z)=z^{-q}+\sum_{n=1-q}^{\infty} a_{n} z^{n} \\ \text { and } \sum_{n=1-q}^{\infty} s_{n}\left|a_{n}-b_{n}\right| \leq \delta\end{array}\right\}$

We have obtained the following result on the $(n, \delta)-$ neighborhoods of analytic

functions of the class $\sum_{\varrho \tau \zeta \xi m p}^{+}(\alpha, \beta, \eta)$.

Theorem 4.2 If the function $g$ as given in Equation (1.7) belonging to the class defined $\sum_{\varrho \tau \zeta \xi k q}^{+}(\alpha, \beta, \eta)$,

I. e. here after it is to be taken as

$N_{\delta}(g) \subset \sum_{\varrho \tau \zeta \xi k q}^{+}(\alpha, \beta, \eta)$,

where it is obviously for all,

$\delta=\frac{2(\tau-\xi)(\tau-\varsigma)+\frac{(\tau-\xi)-\varrho^{2}(\tau-\varsigma)}{\varrho}}{1+2(\tau-\xi)(\tau-\varsigma)+\frac{(\tau-\xi)-\varrho^{2}(\tau-\varsigma)}{\varrho}}$.

From above it is clear that means $\delta$ can't increase.

Proof $\forall g \in \sum_{\varrho \tau \zeta \xi k q}^{+}(\alpha, \beta, \eta)$ of the form (1.7), theorem 3.1 immediately yields

$\sum_{n=1-q}^{\infty} \frac{\{n(\beta+1)+2 q(1-\eta)[1+\beta\langle 4(1-\eta) \alpha-1\rangle]\} \Phi_{n}(\varrho, \tau, \zeta, \xi, k, q)}{2 q(1-\eta) \beta[1+\langle 4(\eta-1) \alpha+1\rangle]} a_{k}$

$\leq \frac{1}{\Phi_{1-q}(\varrho, \tau, \zeta, \xi, 1, q)}$

Let $f(z)=z^{-q}+\sum_{n=1-q}^{\infty} a_{n} z^{n} \in N_{\delta}(f)$

Where $\quad \delta=\frac{2(\tau-\xi)(\tau-\varsigma)+\frac{(\tau-\xi)-\varrho^{2}(\tau-\varsigma)}{\varrho}}{1+2(\tau-\xi)(\tau-\varsigma)+\frac{(\tau-\xi)-\varrho^{2}(\tau-\varsigma)}{\varrho}}>0$.

from the condition (4.10) we have obtained

$\sum_{n=1-q}^{\infty} s_{n}\left|a_{n}-b_{n}\right| \leq \delta$

Using (4.12) \& (4.13), we have obtained

$\sum_{n=1-q}^{\infty} s_{n} a_{n} \leq \sum_{n=1-q}^{\infty} s_{n} b_{n}+\sum_{n=1-q}^{\infty} s_{n}\left|a_{n}-b_{n}\right|$

$\leq \frac{1}{\Phi_{1-q}(\varrho, \tau, \varsigma, \xi, k, q)}+\delta=1$

Thus in accordance with theorem 3.1, we get $f \in$ $\sum_{\varrho \tau \zeta \xi k q}^{+}(\alpha, \beta, \eta)$.To prove more accuracy of functions $g \in \sum_{\varrho \tau \zeta \xi k q}^{+}(\alpha, \beta, \eta)$ and $g \in \sum_{q}^{+}$as follows

$g(z)=$

$z^{-q}+\frac{2 q(1-\eta) \beta[1+\langle 4(\eta-1) \alpha+1\rangle]}{\{\beta[1-2 q(1-\eta)+\langle 4(1-\eta) \alpha-1\rangle 2 q(1-\eta)]+1\} \Phi_{1-q}(\varrho, \tau, \zeta, \xi, k, q)} z^{1-q}$

$g(z)=$

$z^{-q}+$

$\left[\begin{array}{c}\frac{2 q(1-\eta) \beta[1+\langle 4(\eta-1) \propto+1\rangle]}{\{\beta[1-2 q(1-\eta)+\langle 4(1-\eta) \alpha-1\rangle 2 q(1-\eta)]+1\} \Phi_{1-q}(\varrho, \tau, \zeta, \xi, k, q)} \\ +\frac{2 q(1-\eta) \beta[1+\langle 4(\eta-1) \alpha+1\rangle]}{\{\beta[1-2 q(1-\eta)+\langle 4(1-\eta) \alpha-1\rangle 2 q(1-\eta)]+1\} \Phi_{1-q}(\varrho, \tau, \varsigma, \xi, k, q)}\end{array}\right] z^{1-q}$

Hence the function $f \in N_{\delta}(g)$ but in agreement with Theorem 3.1, $g \in \sum_{\varrho \tau \zeta \xi k q}^{+}(\alpha, \beta, \eta)$. Consequently, our theorem is proved. In the next part, we have introduced as given in Equation (1.6)

$k_{k}(z)=$

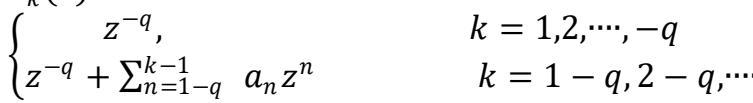

we have determined lower bounds which are sharp for Rep $\left\{\frac{g(z)}{k_{k}(z)}\right\}$ and $\operatorname{Re}\left\{\frac{k_{k}(z)}{g(z)}\right\}$

Theorem 4.3 Let $g \in \sum_{q}$ is given by (1.6) and let $k_{k}(z)$ be given by (4.16). Suppose that

$\sum_{n=1-q}^{\infty} \theta_{n}\left|a_{n}\right| \leq 1$

Here it is obviously for all, $\theta_{n}=\frac{\{n(\beta+1)+2 q(1-\eta)[1+\beta\langle 4(1-\eta) \alpha-1\rangle]\} \Phi_{n}(\varrho, \tau, \zeta, \xi, k, q)}{2 q(1-\eta) \beta[1+\langle 4(\eta-1) \alpha+1\rangle]}$

I. e. here after it is to be taken as $\forall k \geq 1-q$, we get

$\operatorname{Rep}\left\{\frac{g(z)}{k_{k}(z)}\right\}>1-\frac{1}{\theta_{k}}$

$\operatorname{Rep}\left\{\frac{k_{k}(z)}{g(z)}\right\}>\frac{\theta_{k}}{1+\theta_{k}}$

the above defined results are sharp for the following function which is extremal $\forall \mathrm{k} \geq 1-\mathrm{q}$

$$
g(z)=z^{-q}-\frac{1}{\theta_{k}} z^{k}
$$

Proof We can show from the Equation (4.17) that

$\theta_{n+1}>\theta_{n}>1,(n>1-q)$.

hence, by using the hypothesis of the equation (4.17), we get

$\sum_{n=1-q}^{k-1}\left|b_{n}\right|+\theta_{k} \sum_{n=k}^{\infty}\left|b_{n}\right| \leq \sum_{n=1-q}^{\infty} \theta_{n}\left|b_{n}\right| \leq 1$. (4.21)

Let $\quad w(z)=\theta_{k}\left[\frac{g(z)}{k_{k}(z)}-\left(1-\frac{1}{\theta_{k}}\right)\right]=1+$

$\frac{\theta_{k} \sum_{n=k}^{\infty} b_{n} z^{n+q}}{1+\sum_{n=1-q}^{k-1} b_{n} z^{n+q}}$

Using equations (4.21) \& (4.22), we have obtained $\left|\frac{w(z)-1}{w(z)+1}\right|=$ $\left|\frac{\theta_{k} \sum_{n=k}^{\infty} b_{n} z^{n+q}}{2+2 \sum_{n=1-q}^{k-1} b_{n} z^{n+q}+\theta_{k} \sum_{n=k}^{\infty} b_{n} z^{n+q}}\right|$

$\leq \frac{\theta_{k} \sum_{n=1-q}^{k-1}\left|b_{n}\right|}{2-2 \sum_{n=1-q}^{k-1}\left|b_{n}\right|-\theta_{k} \sum_{n=1-q}^{k-1}\left|b_{n}\right|} \leq 1 \quad \forall z$ in $D$.

$\therefore \operatorname{Rep} \mathrm{w}(\mathrm{z})>0,(\forall z$ in D). By using equation (4.22), we can easily obtain equation (4.18). Now $g$ as in (4.20), here after for more accurate results, we can show that for $z \rightarrow 1^{-}$ $\frac{g(z)}{k_{k}(z)}=1-\frac{1}{\theta_{k}} z^{k} \rightarrow 1-\frac{1}{\theta_{k}}$

this proves the bound in the equation (4.18) is the better

possible. Similar to this if Let $\quad \Phi_{z}=(1+$

$\theta k k k(z) g(z)-\theta k 1+\theta k=1-1+\theta k n=k \infty b n z n+q$

$1+n=1-q \infty b n z n+q \quad$ (4.24)

And by making use of equation (4.21), we have obtained the

following relation $\quad\left|\frac{\Phi(z)-1}{\Phi(z)+1}\right|=$

$\left|\frac{-\left(1+\theta_{k}\right) \sum_{n=k}^{\infty} b_{n} z^{n+q}}{2+2 \sum_{n=1-q}^{\infty} b_{n} z^{n+q}-\left(1+\theta_{m}\right) \sum_{k=m}^{\infty} b_{n} z^{n+q}}\right|$

$\leq \frac{\left(1+\theta_{k}\right) \sum_{n=k}^{\infty}\left|b_{n}\right|}{2-2 \sum_{n=1-k}^{\infty}\left|b_{n}\right|+\left(1+\theta_{k}\right) \sum_{n=k}^{\infty}\left|b_{n}\right|} \leq 1$

leads to the Equation given as (4.19). The bound in the Equation (4.19 is more accurate with the extremal function $f$ as shown in Equation (4.20). Our theorem is proved.

References:

[1] Yang, D. G., (1995), on new Subclasses of Meromorphic p-valent functions, J. Mathe. Res. Expo., $15,7-13$.

[2] Ruscheweyh, S., (1981), neighborhoods of univalent functions, proced. American Mathematical Society, 81,521-527.

[3] Srivastava, H. M. and Orhan, H., (2007), coefficient inequality and inclusion relation for some families of analytic and multivalent functions, Appl. Maths. Letters, 20, 686-691.

[4] Uralegaddi, B. A., Somanatha, C., (1991), new criteria for meromorphic starlike univalent functions, bull. Austral. Math Soci., 43, 137-140.

[5] Rajas, S. M. and Khairnar, S. M., (2007), Hadamard product of subclass of univalent functions with +ve coeffs, Int. Jour. of Math. Scie. And Engineering Applications. 1(I), 101-113. 


\section{International Journal of Science and Research (IJSR) \\ ISSN (Online): 2319-7064}

Index Copernicus Value (2013): 6.14 | Impact Factor (2014): 5.611

[6] Raducanu, D., Orhan, H. and deniz, E., (2010), subclasses of Meromorphically multivalent

functions defined by a differential operator, arXiv: 1008.4691v1 [maths. CV]. 1-23.

[7] Orhan, H., (2009), neighborhoods of a certain class of multivalent functions with negative coefficients defined by using a diff operator, Maths. Ineq. Appl., 12(2), 335349.

[8] Clunie, F. R. and Koegh (1960), on starlike and Univalent functions, J. Lond. Mathe. Soci. 35,229-233.

[9] Deniz, E. and Orhan, H., (2010), some properties of certain subclasses of analytic functions with negative coefficients. by using generalized Ruscheweyh derivative Operator, Czechoslovak Math. J., 60(3), 699713.

[10] Dinggong, Y., (1996), on a class of Meromorphic starlike multivalent functions, Bull. Inst. Maths. Acad., Sinica, 24, 151-157.

[11] Goodman, A. W., (1991), on uniformly convex functions, Ann. Polon. Math.56, 87-92. 